\title{
Modified "S" Type Elliptical Excision of a Dermatofibroma
}

\author{
by Jacqueline S. Buchman, DPM ${ }^{1}$, Kelli M. Ashe ${ }^{2}$, Jasmaine N. Shelford $^{3}{ }^{\square}$, Youssef Aoun ${ }^{4}$, \\ Nicholas Noah ${ }^{5}$
}

The Foot and Ankle Online Journal 3 (6): 2

Dermatofibroma is one of the most prevalent non-painful skin tumors, is described as a lesion of increased cells of a hyperplastic manner. This article presents an unusual case of a painful dermatofibroma on the dorsal second metatarsophalangeal joint of the left foot. The challenge of this particular dermatofibroma was the large size and location. Moreover, the potential complication of a dorsiflexed toe from excessive tension at the joint upon closure needed to be avoided. Following investigation of treatment options, the modified " $S$ " type elliptical excision method was most applicable. Neither dermatofibroma's nor a modified " $S$ " type elliptical excision of this lesion is widely mentioned in the literature, therefore this article serves to provide an appropriate method of treatment for large lesions that present along joint spaces.

Key words: Dermatofibroma, "S" type elliptical excision. reproduction in any medium, provided the original work is properly cited. CThe Foot and Ankle Online Journal (www.faoj.org)

$\mathrm{D}$ ermatofibromas are fibrosing cutaneous lesions commonly found on the lower extremities. ${ }^{1}$ There are a number of factors that characterize a lesion as a dermatofibroma. These factors include: 1) An increased number of fibrocytes residing in the dermis and sometimes penetrating into the subcutis; 2) A variable mixture of inflammatory cells; thick unorganized collagen bundles at the periphery; and 3) Hyperplasia of the epidermis and/or melanocytes. $^{2} \quad$ Despite the commonality of these lesions, there is often difficulty in its differentiation from other dermatopathologies and thus diagnosis. Physicians may be misled due to the many clinical and histological variants of dermatofibromas. ${ }^{2}$

Address correspondence to: Barry University School of Podiatric Medicine, 11300 NE $2^{\text {nd }}$ Ave-CCHMM, Miami Shore, FL 33161

Email: drshelford@yahoo.com

1-5 Barry University School of Podiatric Medicine, 11300 NE $2^{\text {nd }}$ AveCCHMM, Miami Shore, FL 33161. (757) 644-7696.
The exact mechanism of the development of these lesions is not known. There is debate whether an inflammatory or neoplastic disorder is the cause. ${ }^{3}$ Many articles support dermatofibromas arising from a reactive inflammatory process. It can be from a minor trauma like an arthropod bite or an internal cause such as a ruptured infundibular cyst. ${ }^{3}$ A neoplastic process is likely due to its clonal proliferative growth perhaps triggered by transforming growth factor-beta or other fibrinogenic factors. ${ }^{4}$

Dermatofibromas are most commonly found on the anterior shins of the lower extremity. ${ }^{1}$ They can develop at any age, but majority occur in young to middle aged females. ${ }^{2}$ Lesions typically present as a single elevated papule or nodule that is well circumscribed and firm, measuring a few millimeters to a few centimeters in diameter. ${ }^{1}$ However, multiple papules or plaques can also occur. ${ }^{3}$ Color of lesions can appear red to red-brown early in presentation and yellow, light brown, or dark brown in older lesions. ${ }^{2,5}$ 


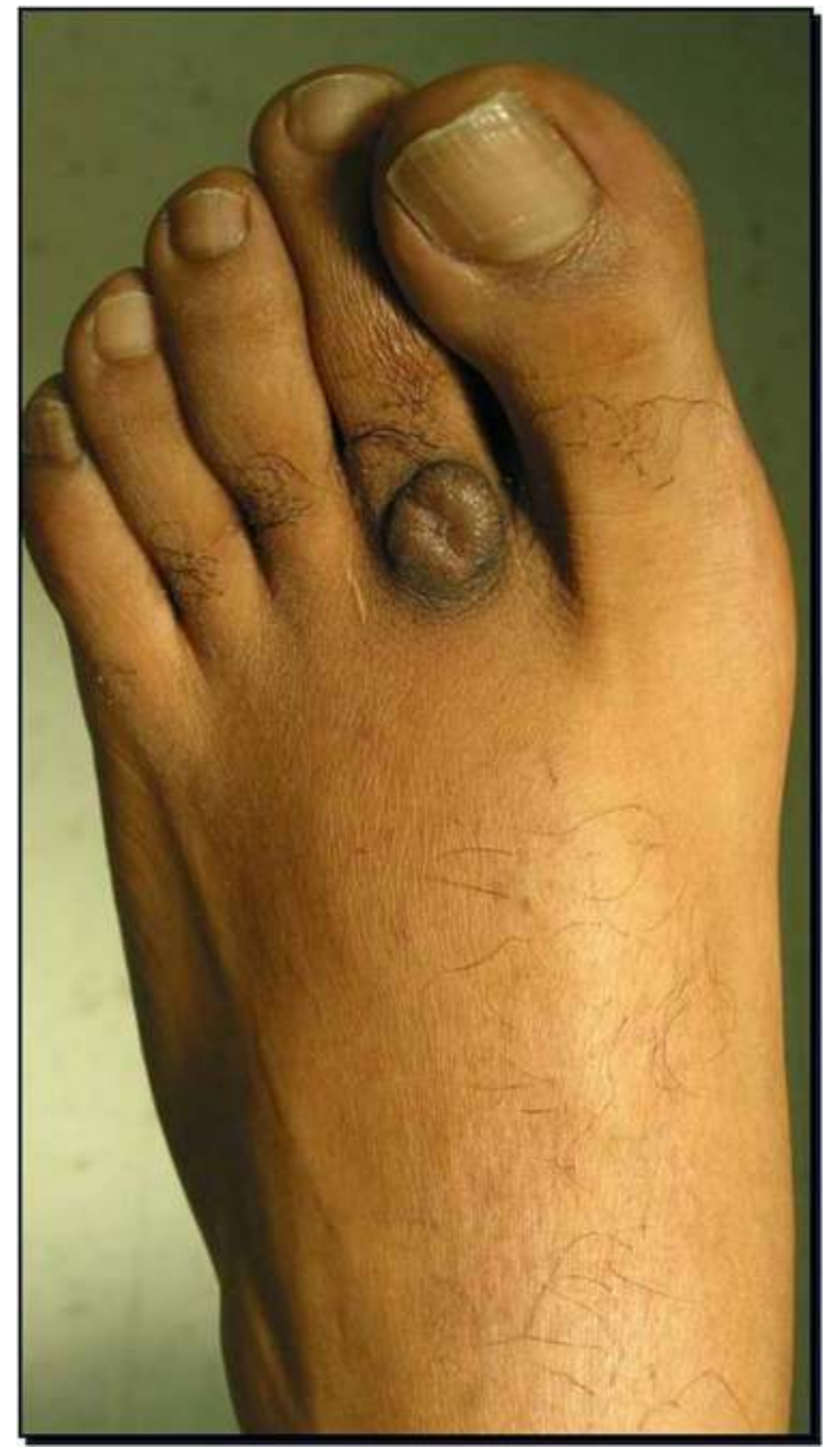

Figure 1 Patient presenting with a hyperpigmented nodule of dorsal second metatarsophalangeal joint.

Tenderness elicited upon palpation and dimpling of the lesion on lateral compression are both clinical signs that can be used to help distinguish the lesion from other dermatopathologies. ${ }^{1}$

The most important in diagnostic distinction that should be made concerning dermatofibromas are distinguishing it from its malignant variants and other aggressive neoplasms, like malignant fibrous histiocytoma, dermatafibromasarcoma protuberans, Kaposi sarcoma, and malignant melanoma. ${ }^{1,2}$ Other differential diagnoses include nodular fasciitis, neurofibroma, leiomyoma, keloid, Spitz nevus, and hemangioma. ${ }^{1,2}$

Symptomatic dermatofibroma's and their excision are not widely present in the literature. This article presents a case of a large, painful dermatofibroma overlying a joint and aims to provide an appropriate treatment method that minimizes potential complications of a hypertrophic scar and decreased range of motion in the joint.

\section{Case Presentation}

A 42 year-old male presented with a complaint of a painful soft tissue mass on the left dorsal aspect of the second metatarsophalangeal joint. The patient reported the lesion slowly developed over four years to its current size; he stated that the pain associated with this lesion was constant and exacerbated when wearing closed-toe shoes. The patient's past medical and social history were essentially unremarkable in relation to the physical examination.

The physical examination revealed a dark brownpigmented nodule without an erythematous base located on the left dorsal aspect of the second metatarsophalangeal joint. (Fig.1)

It measured $12 \mathrm{~mm}$ in diameter and $4 \mathrm{~mm}$ in elevation. Palpation of the lesion and movement through the joint's natural range of motion elicited pain. The remainder of the physical examination findings was unremarkable. Radiographs were taken of the left foot and the radiographic examination revealed no abnormal bony or soft tissue pathologies.

The patient underwent an excisional biopsy of the entire lesion using the modified elliptical excision technique. The procedure was performed under local digital block anesthesia. Incisions were made forming a semi-ellipse superior medial and inferior lateral to the lesion. The ellipse of full-thickness skin and subcutaneous adipose tissue was measured $5.0 \times 1.3 \times$ $0.8 \mathrm{~cm}$. 


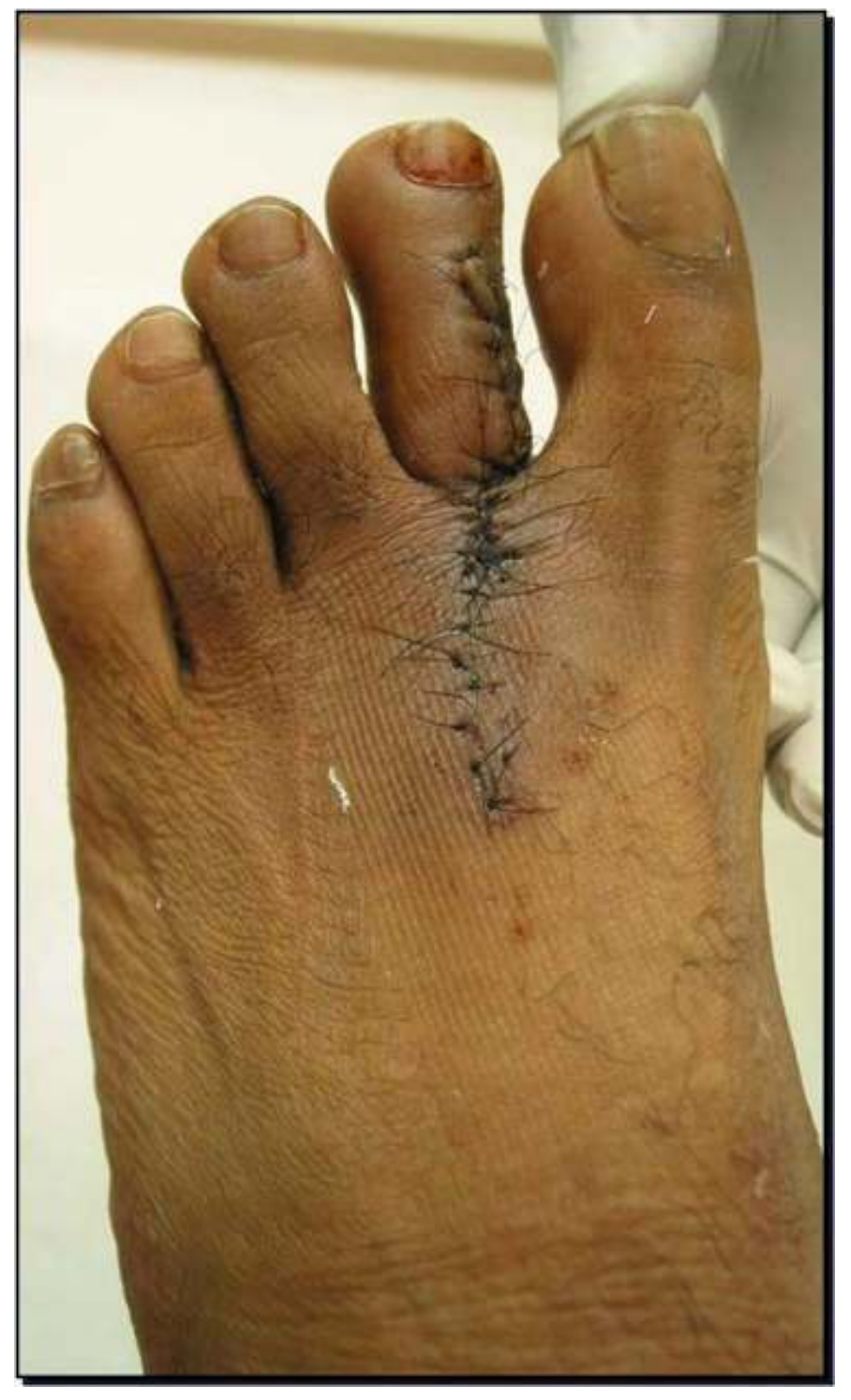

Figure 2 Lazy "S" shaped suture line post-operatively.

Wound closure was performed with 5-0 nonabsorbable nylon sutures leaving a lazy ' $\mathrm{S}$ ' incision. (Fig. 2) Informed consent was obtained from the patient prior to the surgical procedure.

The excised mass was sent to a dermatopathology laboratory. The results of the pathology report revealed an intradermal proliferation of short spindle cells in a storiform growth pattern. (Fig. 3) A definitive diagnosis of dermatofibroma (benign cutaneous fibrous histiocytoma) was established.

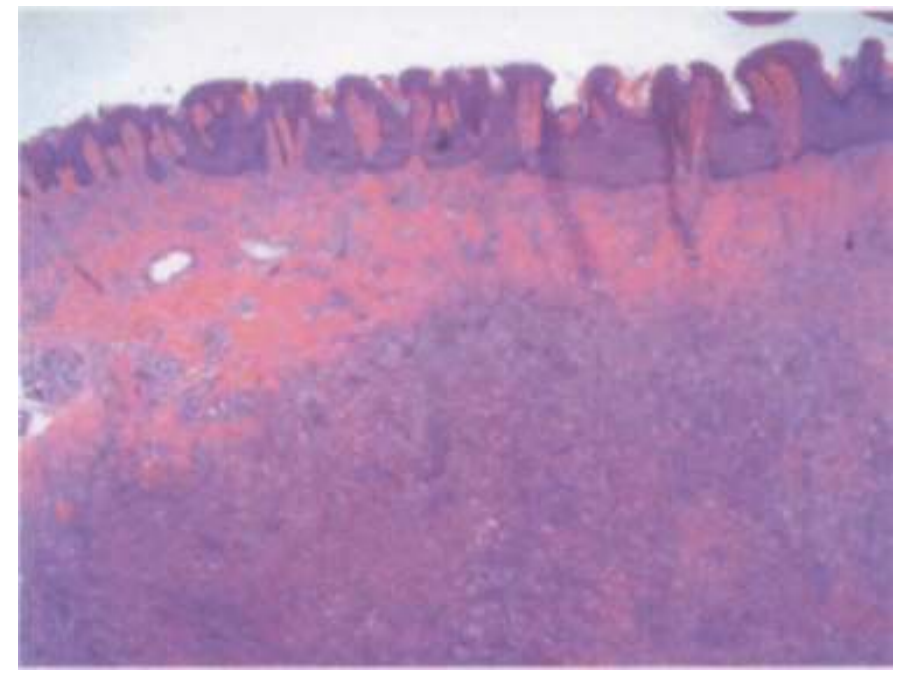

Figure 3 Acanthotic epidermis with hyperpigmented basal keratinocytes over an intradermal proliferation of short spindle cells and enlarged bundles of collagen.

Postoperative visits were routinely performed until complete healing of wound. Sutures were removed at 12 days and the patient was allowed to return to normal shoe gear at that time. The patient was encouraged to perform cross-fiber massage perpendicular to incision scar, to prevent soft tissue adhesions and decrease scar tissue formation. About 2 months post-operatively, patient was instructed to wear Cordran ${ }^{\circledR}$ tape over incision site to help prevent hypertrophic scar formation to left second metatarsophalangeal joint. The patient was followed clinically for 4 months after surgery without any other incident and at last visit there was no contraction or hypertrophy of incision scar.

\section{Surgical Technique}

A modified " $\mathrm{S}$ " type elliptical excision was performed to excise the dermatofibroma. Due to the perpendicular orientation of the excision to the relaxed skin tension lines of the foot and the large dimensions of the lesion, this technique was optimal compared to an elliptical excision. The modified elliptical excision produces a lazy " $S$ " shaped suture line creating less tension across the site facilitating wound healing. 

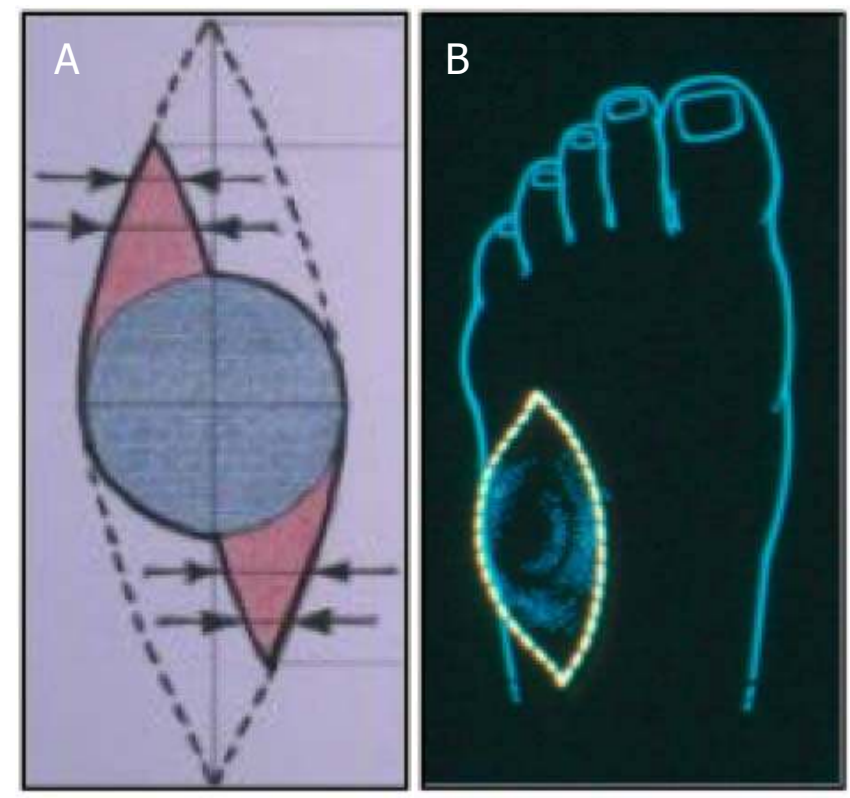

Figure 4 Diagram of S-plasty. (A) Elliptical incision outline. (B)

It is similar to an S-plasty, but with removing the full length of the semi-elliptical skin to form longer, less curved suture line. ${ }^{5}$ (Fig. 4A) A classic elliptical excision would have produced more tension with a vertical suture line. ( Fig. 4B)

The technique began with outlining an ellipse pattern with the recommended length:width ratio of $3: 1{ }^{6}$ A line bisecting the ellipse into two equal halves was then drawn. In order to produce the lazy 'S' suture line, the skin of only the superior medial and the inferior lateral halves of the ellipse were excised along with the dermatofibroma (Fig.5). Following direct closure, a curved suture line was produced running from the medial aspect of the second digit to the lateral proximally of the mid second metatarsal shaft.

This technique has many advantages. These advantages includes: 1) The S-shaped suture line permits less skin tension since incision parallel to RSTL; 2) There is direct closure thus, no delayed healing as when one uses a skin graft; 3) The incision is less likely to produce a hypertrophic scar; 4) There is better aesthetic appearance of the scar; 5) No skin contracture reducing joint range of motion and 6) Good specimen for biopsy. ${ }^{7}$

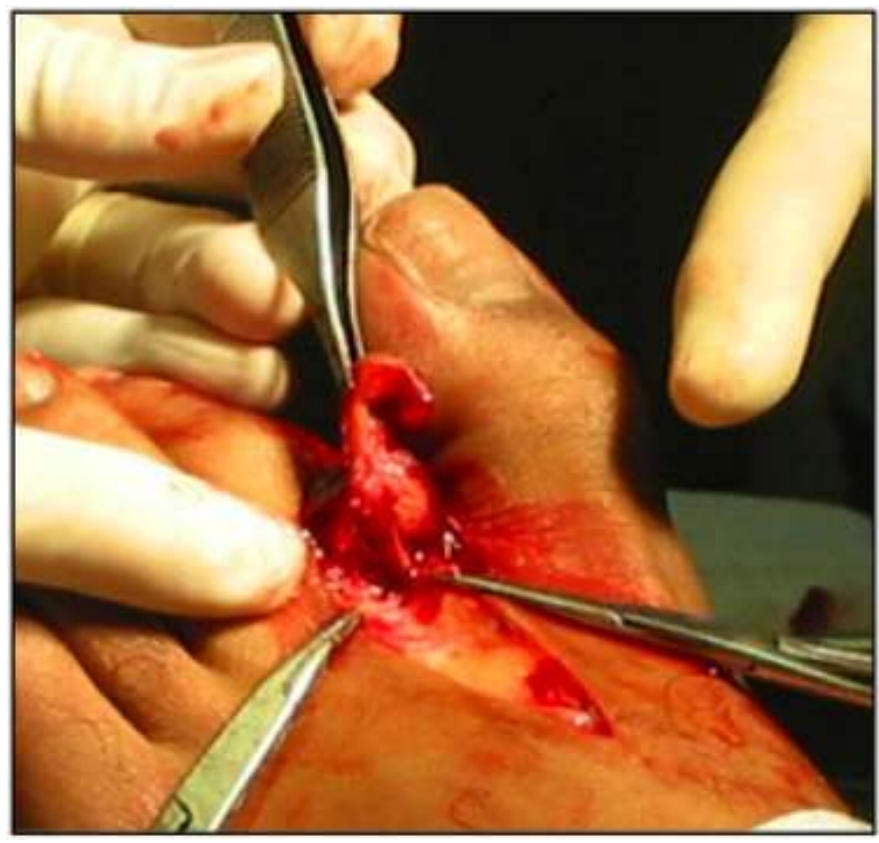

Figure 5 Excision of Dermatofibroma.

\section{Discussion}

Although dermatofibroma's are a very common lesion, it is not a tumor that is reported in the literature often. Few case studies reported on treatment options for dermatofibroma, since treatment is usually not necessary for dermatofibroma. Simple reassurance that the lesion is benign may be indicated encouraging treatment. For any cosmetically unacceptable lesions, lesions that are particularly symptomatic, or for any diagnostic uncertainties, complete excision, including the subcutaneous fat, is the ideal procedure. In this case, the dermatofibroma possesses large dimensions, spanning the width of the base of the second digit and is symptomatic. Choosing an excisional method that would leave a good specimen for biopsy, avoid unreasonable loss of healthy tissue, and result in an aesthetic pleasing scar was the objective.

Some commonly performed techniques were not chosen. Specifically, Weber, et al., stated that an inverted pyramidal biopsy technique might be performed, while still providing adequate tissue for histological evaluation. ${ }^{8}$ 

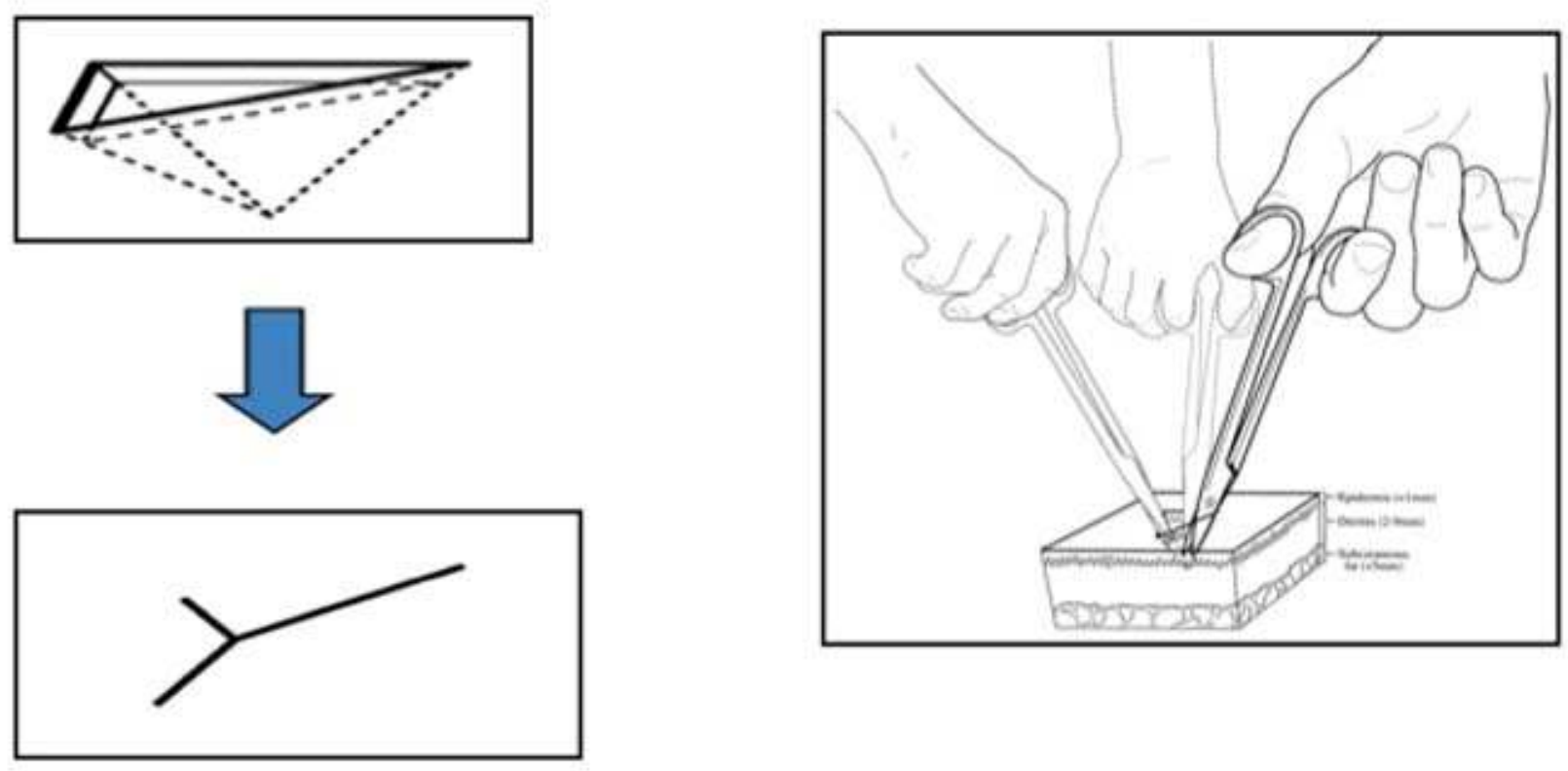

Figure 6 Diagram of inverted pyramid biopsy, final closure, and use of Gradle scissors.

It creates an acute geometric tissue defect with an apex located in the dermis, while leaving a relatively small scar shaped like a Mercedes emblem that is frequently only seen with a hand lens. (Fig 6) This technique however, is not designed to sample large areas of deep dermis or subcutis, which was needed in this case since the lesion grew downward adhering to the extensor digitorium longus. ${ }^{8}$

Cryotherapy is a treatment modality that is commonly used to remove troublesome dermatological lesions. Cryotherapy has advantages as that it typically uses liquid nitrogen to freeze the epidermis and cause necrosis to the pathologic tissue. Painful scar formation can occur if the depth of freezing is not judged properly and in this case the most inferior aspect of the lesion adhered to the extensor digitorium longus. It has been noted in the literature that lesions less than or equal to three centimeters respond best to this form of treatment. ${ }^{\text {? }}$
Cracking of cryolesions does occur typically and is responsible for $8 \%$ of cryotherapy-associated morbidity and can cause excessive bleeding. ${ }^{10}$ Due to the size and location of the lesion in this case, cryotherapy was ruled out as a treatment modality.

Carbon dioxide laser ablation has advantages over other treatments in terms of absence or delay in recurrence due to its influence on fibroblast secretion of growth factor. ${ }^{11}$ Minor complications associated with this form of treatment include hyperpigmentation, acne, rosacea exacerbation, and contact dermatitis. Erythema or hyperpigmentation is typical in colored skin and can lead to anxiety in some patients. The more serious complications that can occur are infection, delayed hypopigmentation, persistent erythema, and prolonged healing. This modality also would rule out the option of a viable tissue specimen for biopsy. ${ }^{12}$ 
With the amount of tissue that had to be removed and the surgical site in close proximity to the extensor digitorium longus, carbon dioxide laser ablation was ruled out to avoid a potential infection which can easily pass along the tendon fascial plane and extended pain and potential damage to the metatarsophalangeal joint. ${ }^{7}$

An elliptical excision was not chosen because it has many disadvantages. Unnecessary healthy skin would have been excised, due to the large size of the dermatofibroma. A full-thickness skin graft would then have been needed to cover the defect. ${ }^{6}$ As previously mentioned, it produces a vertical incision, perpendicular to the relaxed skin tension lines in the foot, which would result in a more prominent scar due to excessive tension at the site. A hypertrophic scar is also likely to form since the incision is directly over the metatarsophalangeal joint. ${ }^{7}$

The modified elliptical excision performed in this case is different from the treatment options mentioned from the reviewed studies above. The ellipse shape is positioned in a way allowing for minimal recurrence, primary healing, decreased skin tension and decreased joint restriction.

\section{Conclusion}

Dermatofibroma's typically present as an asymptomatic, benign, soft tissue papule or nodule measuring a few centimeters at its largest dimensions. ${ }^{1}$ This case presents a unique dermatofibroma that is painful and larger than the norm. Due to its challenging location, lying dorsum the second metatarsophalangeal joint, and its depth, adhering to the extensor digitorum longus tendon, many treatment methods were investigated that would excise the lesion with the least complications. The modified "S" type elliptical excision was determined to best meet our main objectives: obtain a viable specimen for pathology, avoid a hypertrophic scar and decreased joint range of motion, and minimize an unreasonable loss of healthy tissue.
The patient throughout the post-operative course had no complications, healed by primary intention, and resulted in no contraction or hypertrophy of the incision site. Thus, the modified "S" type elliptical excision has proven to be a successful treatment method of excising lesions over the metatarsophalangeal joint.

\section{Acknowledgements}

Special thanks to Gary M. Rothenberg, DPM Attending Podiatrist, Veterans Affairs Medical Center, Miami, Fl for editing the manuscript.

\section{References}

1. Derk F, Nardozza A. Cutaneous fibrous histiocytoma. JAPMA 199787 (5): 241-242.

2. Zelger B, Zelger BG, Burgdorf W. Dermatofibroma - A critical evaluation. Int J surg Pathol 2004 12(4): 333-344.

3. Zelger B, Zelger BG. Dermatofibroma (fibrous

histiocytoma): an inflammatory or neoplastic disorder? Histopathology 2001 38: 379-381.

4. Kubo M, Ihn H, Yamane K, Tamaki K. The expression levels and the differential expression of transforming growth factorbeta receptors in dermatofibroma and dermatofibrosarcoma protuberans. Br J Dermatol 2006 154(5): 919-925.

5. Paolo B, Stefania R, Massimiliano C, Stefano A, Andrea P, Giorgio L. Modified S-Plasty: An alternative to the elliptical excision to reduce the length of suture. Dermatol Surg 2003 24(4): 394-398.

6. Tilleman TR, Neumann M, Smeets N, Tilleman MM. Waste of skin in elliptical excision biopsy of non-melanomatous skin cancer. Scand J Plast Reconstr Surg Hand Surg 2006 40: 352 356.

7. Storm TR, Lee MS. Plastic and Reconstructive Surgery. In: Banks A, Downey M, Martin D, Miller S. McGlamry's

Comprehensive Textbook of Foot and Ankle Surgery. Vol 2, 3 $3^{\text {rd }}$ ed. Philadephia, PA: Lippincott Willaims \& Wilkins; 2001: 14871522.

8. Weber PJ, Moody BR, Foster JA. Inverted pyramidal biopsy. Dermatol Surg 2001 27(7):681-684.

9. Seifert JK, Morris DL. World survey on the complications of hepatic and prostate cryotherapy. World J Surg 1999 23: 109113.

10. Kariappa SM, Morris DL. Cryotherapy - a mature ablation technique. HPB (Oxford). 2006 8(3): 179-118.

11. Krupa Shankar DS, Kushalappa AA, Suma KS, Pai SA. Multiple dermatofibromas on face treated with carbon dioxide laser. Indian J Dermatol Venereol Leprol 2007 73(3): 194-195.

12. Krupa Shankar DS, Chakravarthi M, Shilpakar R. Carbon dioxide laser guidelines. J of Cutaneous and Aesthetic Surgery 2009 2(2): 72-80. 